\title{
Panteles zōion e pantelōs on: Vita, anima e movimento intellegibile nel Timeo (e nel Sofista)
}

\author{
Francesco Fronterotta
}

\begin{abstract}
In this article, I try to propose some reflections about the nature and status of the intelligible in the Timaeus, particularly with respect to its features of a properly being and above all vital reality. The attribution of "life" and "vitality" to the intelligible certainly has an analogical character, that is, it depends on the consideration of the sensible: since the cosmos is a sensible living being and is a copy of an intelligible model, then the intelligible model must be configured as an intelligible living being. Now, to be "living", for a sensible reality, means to have a soul that animates a body, that is a soul which is embodied; but this seems to apply only in the case of the sensible. What does it mean, then, and what does it entail, to be "living" for an intelligible reality? Some interpretative hypotheses on this point are examined here and a possible overall explanation is suggested.
\end{abstract}

\section{Keywords}

Plato - Timaeus - intelligible (being) - life - soul - intellect

Per tentare di svolgere qualche considerazione intorno alla natura e allo statuto dell'intellegibile nel Timeo $^{1}$, particolarmente rispetto ai suoi tratti di realtà propriamente essente e vitale, è necessario prendere le mosse dalla ben nota distinzione, posta in $27 \mathrm{~d}-28 \mathrm{~b}$, fra « ciò che sempre è, senza avere genera-

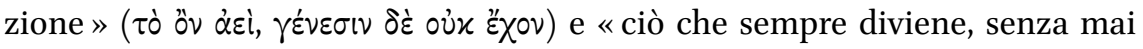

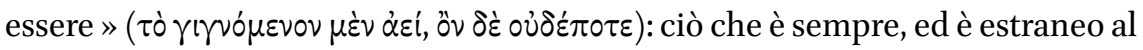
divenire, « si coglie con il pensiero e se ne può rendere conto razionalmente » (

1 Per una presentazione introduttiva che fornisce le coordinate d'insieme che presiedono all'esposizione di Timeo, e per l'indicazione della bibliografia pertinente, sia lecito rinviare alla mia Introduzione a Fronterotta, ed., Platone, Timeo, 23-35, cui farò nuovamente riferimento in seguito. 
è « oggetto dell'opinione che deriva dalla sensazione di cui non si può rendere

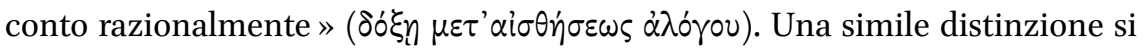
basa sul fatto che, mentre ciò che è davvero, mai divenendo, resta immobile e immutabile ( $\dot{\alpha} \varepsilon \dot{\imath} \varkappa \alpha \tau \dot{\alpha} \tau \alpha \dot{\tau} \tau \dot{\alpha}$ őv) e può perciò costituire l'oggetto di una conoscenza vera e a sua volta immutabile, ciò che diviene, invece, mai essendo dav-

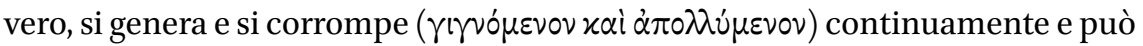
costituire soltanto l'oggetto di una conoscenza incerta, a sua volta mutevole e corruttibile, l'opinione ${ }^{2}$.

Fra gli ambiti dell'essere e del divenire sussiste inoltre una duplice relazione causale, perché l'intera sfera di ciò che diviene, in quanto è caratterizzata da generazione e corruzione, suppone l'intervento di una causa a partire da cui ap-

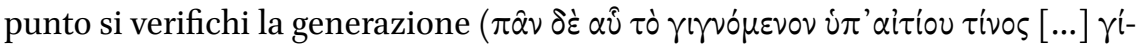
$\gamma v \varepsilon \sigma \theta \alpha$ ) - una causa efficiente, quindi, giacché è « impossibile, per qualunque

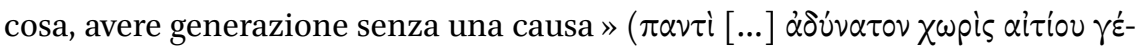
$\nu \varepsilon \sigma(\nu \sigma \chi \varepsilon \hat{\imath}$ ), i cui tratti propriamente produttivi inducono ad associarla a una funzione artigianale e demiurgica - e l'esistenza di un modello in conformità al quale la realtà generata sia costituita nella sua forma e nelle sue proprietà

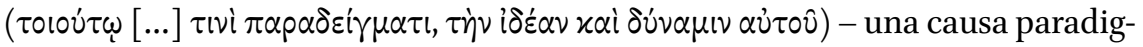
matica, dunque, che, se coincide con l'essere immobile e immutabile ( $\tau \dot{o} x \alpha \tau \dot{\alpha}$ $\tau \alpha u ̉ \tau \dot{\alpha}$ É $\chi \circ v)$, conduce alla riproduzione, a partire da se stessa, di copie o immagini belle, nella misura in cui conservano traccia della perfezione del modello; mentre, se tale causa paradigmatica coincidesse con un'altra realtà generata, le copie o immagini riprodotte a partire da questa non potrebbero che rispecchiare l'imperfezione del modello nella loro estraneità alla bellezza ${ }^{3}$. È chiaro come causa efficiente e causa paradigmatica - il demiurgo e il modello, ho

2 Si tratta del caratteristico principio, onnipresente nei dialoghi platonici, che stabilisce la corrispondenza fra l'ambito epistemico delle diverse forme e facoltà della conoscenza e l'ambito ontologico dei diversi oggetti che esse assumono come proprio contenuto, in virtù della quale natura e grado di verità della conoscenza dipendono dallo statuto dei suoi oggetti. Devo rinviare, per una formulazione generale della questione e per una discussione della

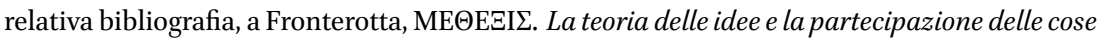
empiriche. Dai dialoghi giovanili al Parmenide, specie 62-79; e all'acuta disamina di Leszl, « Ragioni per postulare idee».

3 Sulla natura di tale relazione causale e sulle diverse proposte esegetiche intorno alla natura della causalità, formale, paradigmatica o propriamente efficiente, delle idee intellegibili rispetto alle cose sensibili, rinvio a una serie di lavori recenti che hanno significativamente rinnovato il panorama degli studi platonici: Sedley, «Platonic Causes »; Natali, « La forma platonica è una causa formale?»; Ferrari, «Questioni eidetiche»; Fronterotta, «Chiusura causale della fisica e razionalità del tutto : alcune opzioni esegetiche sull'efficienza causale delle idee platoniche »; e, con particolare riferimento al Timeo, Fronterotta, «Modello, copia, ricettacolo : monismo, dualismo o triade di principi nel Timeo?». 
dèmiourgos e to paradeigma - siano strettamente connesse, dal momento che l'azione produttiva del demiurgo, che innesca il processo della generazione fra le realtà in divenire, si basa e dipende da un modello, appunto, da riprodurre nelle sue copie o immagini; come pure la funzione paradigmatica del modello, perché possa realizzarsi nella riproduzione delle sue copie o immagini, esige l'intervento "operativo" di un agente, senza il quale rimarrebbe inerte da un punto di vista produttivo, appunto come un modello che attenda di essere riprodotto. Dei diversi aspetti che il semplice richiamo di questo passo evoca desidero porne in rilievo soltanto tre, in forma puramente generale: (1) nessuna generazione è possibile se non in seguito a, e in conseguenza di, un'azione causale; (2) ogni generazione consiste per necessità nella riproduzione di un modello; (3) il modello si configura come una causa paradigmatica, e non efficiente, della generazione, perché la causa efficiente della generazione, benché indubbiamente connessa al modello, ne è altrettanto evidentemente dissociata, se viene posta l'alternativa relativa al genere di modello, eterno o generato, assunto e riprodotto nell'atto generativo vero e proprio ${ }^{4}$.

Questo schema ontologico, applicato all'esame del cosmo (28b-29b), della sua natura e della sua struttura, porta a riconoscere che il nostro mondo, in quanto è di natura sensibile e perciò soggetto al divenire e alla trasformazione, fa parte delle realtà caratterizzate da generazione e corruzione $\left(\tau \dot{\alpha} \delta^{\prime} \alpha \hat{l}-\right.$

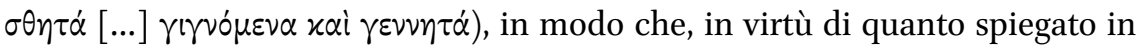
precedenza, deve possedere un principio e una causa della generazione ( $\dot{\alpha} \pi^{\prime} \dot{\alpha} p$ -

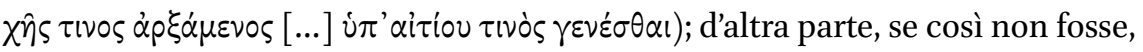
il cosmo si rivelerebbe esso stesso, in quanto privo di generazione e corruzione, eterno e finirebbe per coincidere allora con il modello intellegibile, sicché il riconoscimento che vi è un ambito di realtà in divenire impone la postulazione di un ambito di realtà eterne come suo modello: rimane controverso, ma non intendo toccare qui questo punto, se l'ammissione del carattere diveniente del mondo implichi che esso ha avuto un inizio e un'origine nel tempo, e sia stato dunque effettivamente generato, o semplicemente che è di natura sensibile, e sia dunque affetto nel suo complesso ed eternamente da generazione e

4 In favore dell'interpretazione opposta, di un'identificazione della causa paradigmatica rappresentata dal modello intellegibile e della causa efficiente associata al demiurgo, si è espresso Ferrari, «Causa paradigmatica e causa efficiente: il ruolo delle idee nel Timeo », e «Der entmythologisierte Demiurg», riprendendo e ampiamente sviluppando alcuni spunti suggeriti, fra gli altri, da Perl, «The Demiurge and the Forms. A Return to the Ancient Interpretation of Plato's Timaeus». Ho discusso, e in certa misura criticato, questa proposta esegetica nell'articolo «Questioni eidetiche in Platone: il sensibile e il demiurgo, l'essere e il bene», 421-424. 
corruzione rispetto alle cose in esso contenute ${ }^{5}$. Ed è in base a tali premesse che Timeo richiama nuovamente, come «costruttore e padre $(\pi 0 i \eta t \eta \dot{\nu}$ xai

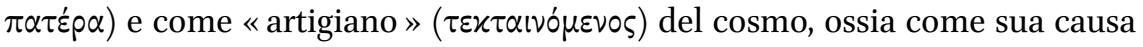
produttiva o efficiente, il demiurgo; e come modello in conformità al quale si realizza l'attività produttiva di quest'ultimo ( $\pi$ pòs $\pi$ ó $\tau \varepsilon p o \nu ~ \tau \hat{\omega} \nu \pi \alpha \rho \alpha \delta \varepsilon r \gamma \mu \alpha \dot{\alpha} \tau \omega \nu$ [...] a

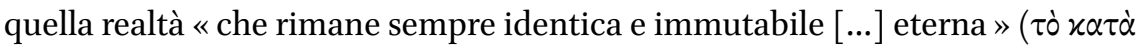

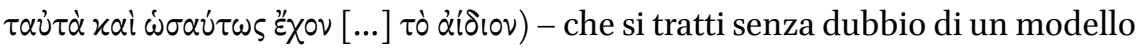
appartenente all'ambito di « ciò che sempre è, senza avere generazione » è reso certo dalla semplice constatazione della bellezza del cosmo che, come già sappiamo, rinvia di necessità alla perfezione di un paradigma eterno a partire dal quale è stato riprodotto, e dall'assunto indiscusso della bontà del demiurgo,

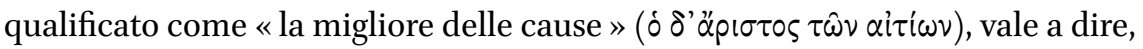
presumibilmente, la più compiutamente efficace ${ }^{6}$. Se ne conclude così che il cosmo non è altro che un'immagine generata di un modello eterno - immagine e modello costituendo due generi da tenere fra loro opportunamente distinti

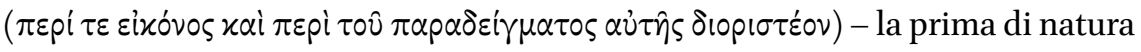
sensibile, il secondo che «si coglie con il ragionamento e con il pensiero » ( $\tau \dot{0}$

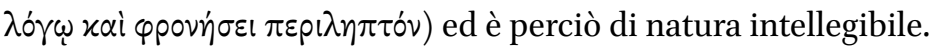

A tale scansione Timeo non esita a ricondurre anche l'ambito dei logoi: come fra le cose che sono e fra i modi di conoscenza che a esse si rivolgono,

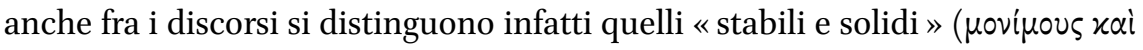
$\dot{\alpha} \mu \varepsilon \tau \alpha \tau \tau \dot{\omega} \tau o v \varsigma)$, quando riguardano un contenuto a loro volta «stabile, saldo

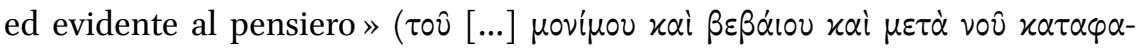
voûs), cioè la realtà intellegibile che è sempre, e quelli soltanto «verosimili » 0

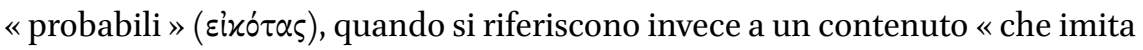

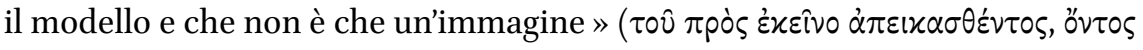
$\delta \varepsilon \grave{~ \varepsilon i x o ́ v o s), ~ q u i n d i ~ a l l a ~ r e a l t a ̀ ~ s e n s i b i l e ~ i n ~ d i v e n i r e ~ c h e, ~ i n ~ q u a n t o ~ c o p i a ~ o ~ i m i-~}$ tazione della prima, è solo simile, ma non identica, a essa. Donde la ben nota

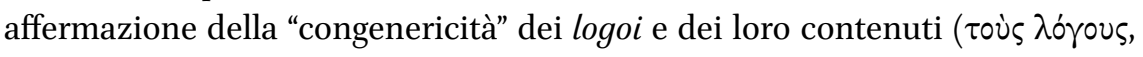

5 Non esamino dunque qui la delicatissima questione dell'inizio nel tempo della vicenda cosmica o della sua eternità, come risposta alla domanda esplicitamente posta da Timeo $(28 \mathrm{~b})$ « se [il mondo] sia sempre stato, senza avere né principio né generazione, oppure se sia stato

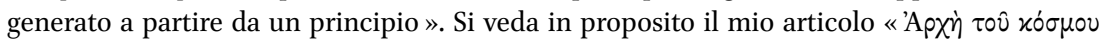

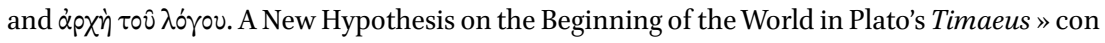
gli opportuni riferimenti bibliografici.

6 Pure da parte rimane in questa sede la questione della "bellezza" del cosmo generato e della "bontà" del demiurgo che lo ha prodotto, che fissa i termini della prospettiva rigorosamente teleologica sottesa alla cosmologia del Timeo. Cfr. ancora Fronterotta, ed., Platone, Timeo, $85^{-88 .}$ 


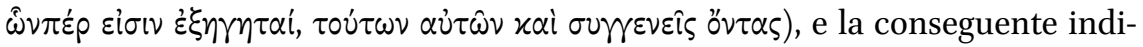
cazione di una corrispondenza fra essere e verità delle cose che sono e dei discorsi pienamente scientifici che ne parlano e fra divenire e semplice verosimiglianza delle cose soggette a generazione e corruzione e dei discorsi solo opinativi a esse relativi $(29 \mathrm{~b}-\mathrm{d})^{7}$.

Più avanti, il dialogo torna a più riprese, per precisarla e articolarla ulteriormente, su questa generale scansione onto-cosmologica. In 48e-49a, Timeo precisa che, in aggiunta ai duo eide individuati in precedenza, «l'uno posto come genere del modello, intellegibile e sempre identico a se stesso » ( $\tilde{v} \nu \mu \dot{\varepsilon} \nu$

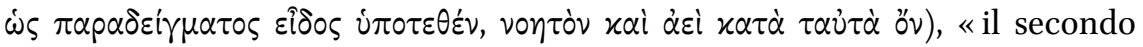
come imitazione del modello, soggetto a generazione e visibile» ( $\mu \dot{i} \mu \eta \mu \alpha \delta \dot{\varepsilon}$

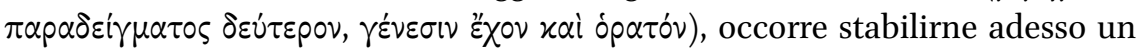
terzo, che prima non era apparso necessario e che risulta «difficile e oscuro »

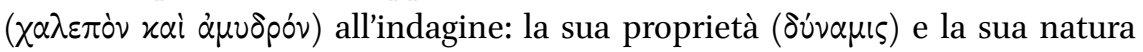
( $\varphi \dot{\sigma} \sigma \varsigma)$ sembrano consistere essenzialmente nel ruolo che a tale genere spetta

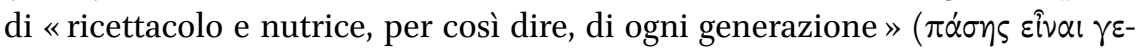

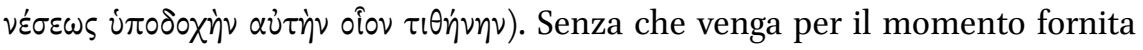
nessuna ulteriore delucidazione, pare però plausibile intendere questa duplice denominazione del terzo genere nel senso che esso accoglie, come "ricettacolo", e alimenta, come "nutrice", ogni processo produttivo, cioè precisamente ogni processo che, a partire dal primo genere, il modello intellegibile, conduce alla realizzazione del secondo genere, che consta delle imitazioni, appunto soggette al divenire, alla generazione e alla corruzione, del modello intellegibile; in tal senso, il terzo genere sembra prestare un fondamento "spaziale" o "locale" e a un tempo "materiale" o "sostanziale" alla generazione del sensibile a imitazione dell'intellegibile ${ }^{8}$. Poco oltre, in $50 \mathrm{oc}-\mathrm{d}$, questo schema triadico risulta confermato, perché si indicano ancora tre generi da tenere a mente

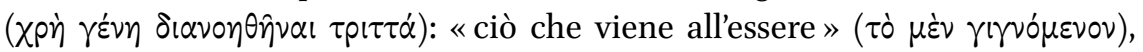
che corrisponde evidentemente al secondo genere del passo precedente, alla

7 Un articolato esame dei diversi livelli di "verità" e "verosimiglianza" del discorso di Timeo è stato condotto da Donini, «Il Timeo: unità del dialogo, verosimiglianza del discorso », specie $37-5$.

8 Dell'ampissima serie di studi relativi alla natura e alla funzione onto-cosmologiche della chōra, di cui è impossibile trattare qui, ricordo soltanto alcuni indispensabili punti di riferimento recenti, che danno conto anche del dibattito critico pertinente: si vedano Algra, Concepts of Space in Greek Thought, 74-120; Miller, The Third Kind in Plato's Timaeus; e i più agili lavori di Ferrari, «La chora nel Timeo di Platone. Riflessioni su "materia" e "spazio" nell'ontologia del mondo fenomenico », e di Brisson, « La matière chez Platon et dans la tradition platonicienne ». Ho a mia volta esaminato e discusso i tratti "spazio-materiali" della chōra appena evocati nell'articolo « Luogo, spazio e sostrato "spazio-materiale" nel Timeo di Platone e nei commenti al Timeo». 
realtà sensibile in divenire soggetta a generazione e corruzione, paragonato a una «natura intermedia » fra gli altri due generi ( $\tau \dot{\eta} \nu \delta \dot{\varepsilon} \mu \varepsilon \tau \alpha \xi \dot{\nu} \tau o u ́ \tau \omega \nu ~ \varphi v ́ \sigma v)$ e come « a un figlio » ( $\dot{x} x \gamma o ́ v \omega)$ di quelli; « ciò in cui viene all'essere [scil., ciò che

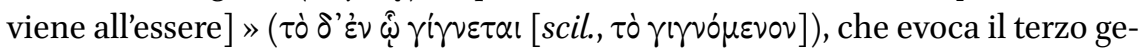
nere, in quanto "spazio" o "luogo" della generazione del secondo genere, ossia

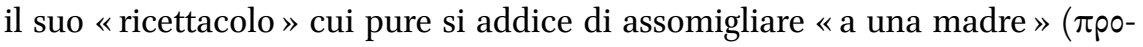
$\sigma \varepsilon i x \alpha \dot{\alpha} \sigma \alpha l$... $\tau \dot{\partial} \mu \dot{\varepsilon} \nu \mu \eta \tau \rho i ́)$, così richiamandone nuovamente la funzione "materiale" o "sostanziale"; e " ciò a somiglianza di cui viene all'essere ciò che viene

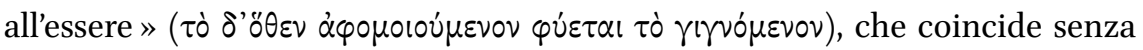
dubbio con il primo genere, il «modello » intellegibile a imitazione del quale

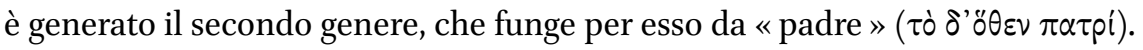
Ancora, in $51 \mathrm{e}-52 \mathrm{c}$, dopo aver rievocato la distinzione tracciata fin da $27 \mathrm{~d}-28 \mathrm{~b}$ fra realtà intellegibili e sensibili, le prime, le idee, oggetto stabile e immutabile del pensiero, le seconde, le cose che percepiamo tramite i sensi, contenuto variabile e mutevole dell'opinione, presentate come duo gene fra loro alternativi, Timeo ribadisce la sua posizione, articolandone i dettagli: bisogna ammettere un primo genere, che è «sempre identico, ingenerato e incorruttibile »

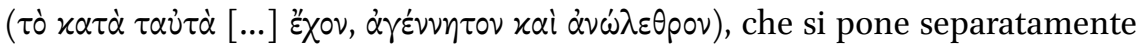
da qualunque altra cosa, che è invisibile e impercettibile, ma si coglie con il

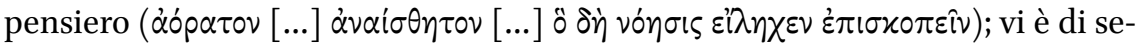
guito un secondo genere, che « ha lo stesso nome ed è simile al primo » ( $\tau \dot{\delta} \delta \dot{\varepsilon}$

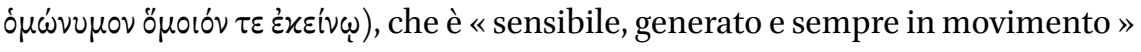

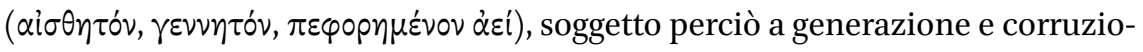

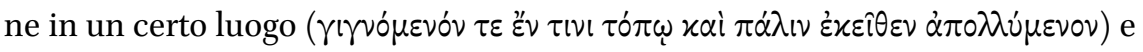
contenuto dell'opinione che si accompagna alla percezione ( $\delta \delta^{\prime} \xi_{\eta} \mu \varepsilon \tau$ ' $\alpha i \sigma \theta \dot{\eta} \sigma \varepsilon-$ $\omega \varsigma \pi \varepsilon p\left(\lambda \eta \pi \tau \tau^{\prime} v\right)$; e vi è infine un terzo genere, cui è attribuita qui la denomina-

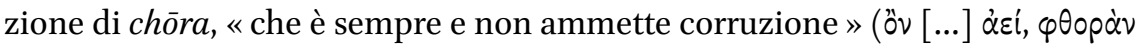
o $\pi_{0}$ fra ragionamento e percezione sensibile e a un tempo estraneo a entrambi, risulta apparentato al sogno e alla sua debole credibilità, e che, ciononostante, fornisce un luogo o una sede ( $\varepsilon^{\prime} \delta \rho \alpha \nu ~ \delta \dot{\varepsilon} \pi \alpha \rho \dot{\varepsilon} \chi 0 \nu$ ) per la generazione di ogni

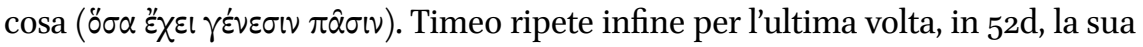

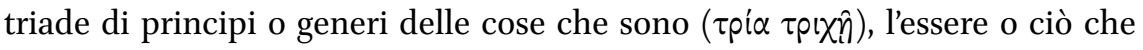

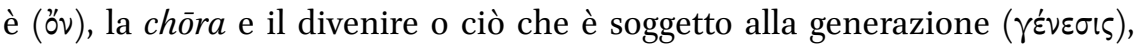
che precedono la costituzione del mondo, posti in un rapporto di collaborazione in base al quale la chōra, come «nutrice della generazione» ( $\gamma \varepsilon v \varepsilon \dot{\sigma \varepsilon \omega \varsigma}$ $\tau \theta \dot{\eta} \nu \eta \nu)$, è per ciò stesso naturalmente disposta ad accogliere forme e figure

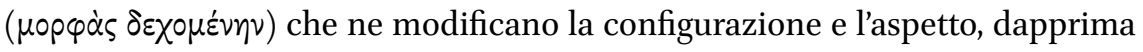
secondo un andamento conflittuale e disordinato, in seguito, verosimilmente per l'intervento della divinità demiurgica, a imitazione delle realtà intellegibili, 
cioè del modello eterno, dando luogo così alla generazione del cosmo, appunto in virtù dell'interazione fra il modello e la chōra operata dall'attività ordinatrice della divinità demiurgica $\left(53^{\mathrm{a}-\mathrm{b}}\right)$.

Attenendomi ai miei scopi attuali, lascerò del tutto da parte la temibile questione delle effettive modalità della generazione del cosmo sensibile e, di conseguenza, dell'interpretazione, pure assai controversa, della figura e del ruolo operativo del demiurgo, come anche, d'altro canto, l'altrettanto spinoso problema dello statuto ontologico e della natura funzionale della chōra ${ }^{9}$; trarrò invece dalla sintetica ricognizione delle successive prese di posizione di Timeo appena tratteggiata le indicazioni che mi appaiono pertinenti in relazione al tema annunciato della descrizione dei tratti costitutivi del modello intellegibile come realtà propriamente essente e vitale. Abbiamo appreso infatti (fin da $27 \mathrm{~d}-28 \mathrm{a}$, ma cfr. pure $48 \mathrm{e}-49 \mathrm{a}$ e $5 \mathrm{~b}-52 \mathrm{a}$ ) che le idee intellegibili, che compongono nella loro pluralità e totalità il modello eterno, si pongono senza dubbio come principi appartenenti all'essere che è sempre, esente da genera-

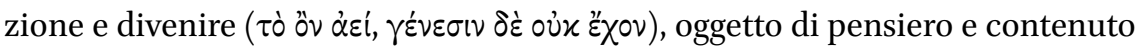
di ragionamento ( $\varkappa \alpha \tau \dot{\alpha} \tau \alpha \dot{\tau} \tau \dot{\alpha} \partial ̋$ ); di esse il demiurgo si serve come esemplari a partire da cui produrre il mondo sensibile (28b-29a), sicché, appunto in quanto modelli, si trovano paragonate a un "padre" che, esercitando una causalità paradigmatica sul materiale rappresentato dall'elemento materno, definisce la forma del figlio e contribuisce in tale misura alla sua generazione $(5 \circ)^{10}$, per essere ancora concepite in seguito alla stregua di schemi formali e numerici di cui la divinità fa uso nella sua opera di ordinamento cosmico $(53 \mathrm{~b})$; le idee sono dotate infine $(51 b-c)$ di piena autonomia e auto-sufficienza ontologica perché tutte

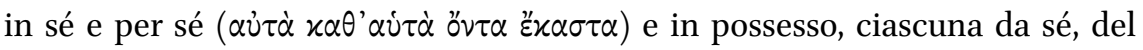

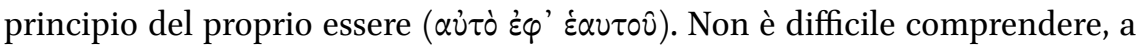
simili condizioni, per quale ragione l'intellegibile nel suo complesso occupi il rango ontologico più elevato in questa gerarchia del reale, configurandosi così

9 Rinvio rispettivamente, per questi aspetti, a Brisson, Le même et l'autre dans la structure ontologique du Timée de Platon, 55-101, che fornisce tuttora un eccellente punto di partenza storico e filosofico per l'esame della figura del demiurgo del Timeo, e ai lavori citati nella nota precedente.

10 Vale la pena notare, pur senza poterne approfondire qui le eventuali implicazioni, che la metafora del "padre", che nei passi citati del Timeo allude indubbiamente a una forma di causalità paradigmatica, cioè all'esercizio della funzione di un modello cui il figlio assomiglia riproducendone i tratti nell'elemento materiale e ricettivo materno rappresentato dalla chōra, è invece utilizzata altrove nei dialoghi platonici (come del resto nel corpus aristotelico) come esemplificazione di una forma di causalità propriamente efficiente $\mathrm{e}$ produttiva (cfr. per esempio Hipp. Ma. 297b-c), tale per cui il "padre" simboleggia il principio attivo della generazione del figlio. 
a pieno titolo, in quanto appunto propriamente essente, come modello eterno della generazione del cosmo sensibile.

Ora, tornando alla sezione del dialogo da cui ho preso le mosse, la causa

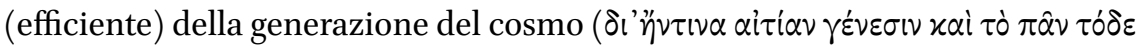

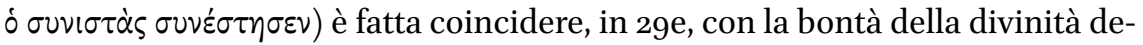
miurgica che, appunto in quanto buona, cioè priva di invidia ( $\alpha \gamma \alpha \theta \dot{\rho} \varsigma \hat{\eta} v, \dot{\alpha} \gamma \alpha \theta \hat{\omega}$

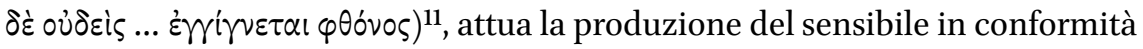
alla propria bontà, vale a dire aspirando a rendere il proprio prodotto "buono"

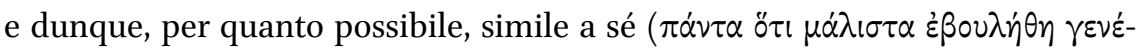

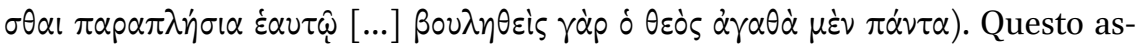
sunto si traduce immediatamente in tre linee-guida dell'azione produttiva del demiurgo, che consiste (1) nell'attribuzione di un ordine al movimento disordinato che appartiene allo stato pre-cosmico, « considerando che questo è in

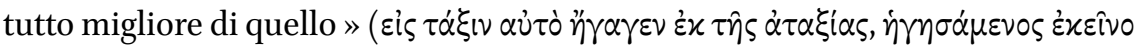

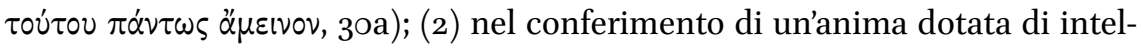

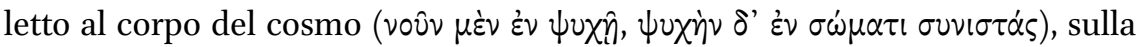
base dell'argomento che « dalle cose che sono per loro natura visibili » ( $\dot{\varepsilon} \chi \tau \hat{\omega} \nu$ $\varkappa \alpha \tau \dot{\alpha} \varphi v ́ \sigma \nu$ óp $\alpha \tau \hat{\omega} \nu$ ) è possibile generare un essere migliore rispetto a uno peg-

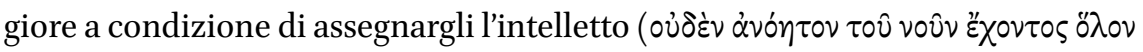

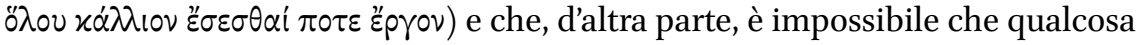

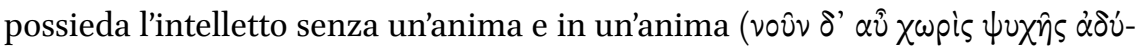

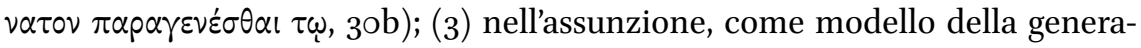

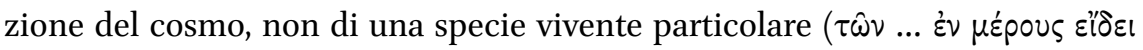
$\pi \varepsilon \varphi v x o ́ \tau \omega \nu$ ), perché «nulla che assomigli a un essere incompleto potrebbe mai

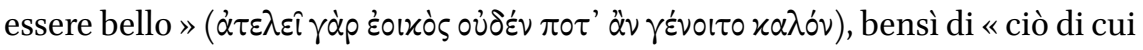
fanno parte gli altri viventi, singolarmente o secondo la specie, a questo, fra

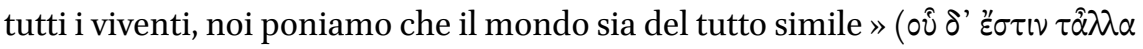

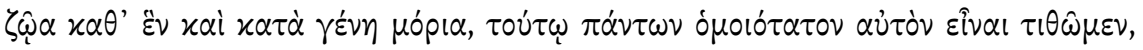
3oc). Lasciando da parte il primo di questi criteri operativi, che ha carattere piuttosto generale, è interessante osservare come il secondo di essi implichi che la questione della presenza dell'anima e dunque dell'animazione vitale

11 Il termine greco phthonos, letteralmente "invidia" o "gelosia", designa, per esempio, l'atteggiamento della divinità nei confronti dell'uomo che oltrepassa, a qualunque titolo, i limiti della propria natura, per avvicinarsi alla condizione divina. In questo caso, la divinità diviene "invidiosa" o "gelosa" dell'uomo e procede alla sua punizione, facendolo cadere in errore o ingannandolo in qualche modo, per esempio accecando i suoi occhi e la sua mente. È da tale punto di vista che il demiurgo, non essendo per nulla "invidioso" o "geloso", non intende privare il cosmo prodotto dalla sua azione generativa di nessuna delle caratteristiche che possano renderlo "buono", cioè, per quanto possibile, simile a sé. Si veda in proposito Brisson, « La notion de phtónos chez Platon ». 
riguarda esclusivamente le realtà sensibili dotate di corpo: è in relazione alle « cose che sono per loro natura visibili», infatti, che ha senso chiedersi quale sia l'apporto che al loro statuto viene dall'attribuzione del nous e dell'anima, se il nous non può sussistere, per definizione, dissociato da quella; ed è appunto

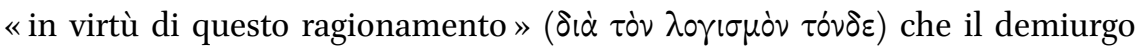
procede alla costituzione del cosmo ponendo il nous nell'anima e l'anima nel corpo - si tratta evidentemente qui dell'anima e del corpo del mondo - tale duplice innesto, dell'intelletto nell'anima e dell'anima nel corpo, che conduce a concepire il cosmo generato come « un vivente dotato di anima e di intelletto »

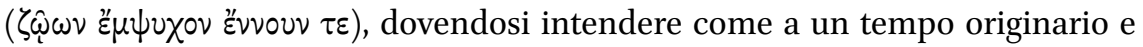
strutturale, vale a dire come assolutamente necessario e universalmente vero: se l'associazione dell'intelletto all'anima e dell'anima al corpo è parte del disegno demiurgico, comunque lo si interpreti, ciò comporta senza alcun dubbio che nessun intelletto può sussistere indipendentemente da un'anima né nessun'anima indipendentemente da un corpo, cioè al di fuori dell'ambito delle cose sensibili ossia, ancora, nell'intellegibile. E del resto, se all'anima spetta di "animare" un corpo e se all'intelletto compete di rendere migliore un essere che potrebbe, in sua assenza, essere peggiore, quale contributo offrirebbero l'anima e l'intelletto in un ambito rispettivamente già perfetto e compiuto e privo di corpo, come è di per sé l'intellegibile?

Ciò non impedisce che, pur rinunciando a ogni riferimento all'anima e al corpo, venga ammessa una forma di "vitalità" dell'intellegibile, come risulta chiaramente dall'assunzione del terzo criterio operativo dell'azione produttiva demiurgica, se il modello eterno cui essa si ispira consiste nella totalità dei viventi e si configura quindi esso stesso come quello, fra i viventi, che tutti li comprende. Infatti, è proprio a questo punto che il tratto "vitale" dell'intellegibile si trova esplicitato: innanzitutto (30c), il modello è detto includere « tutti i viventi intellegibili » ( $\tau \dot{\alpha} \nu \circ \eta \tau \dot{\alpha} \zeta \hat{\omega} \alpha \pi \dot{\alpha} \nu \tau \alpha)$ proprio come il cosmo sensibile contiene

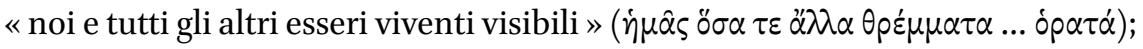
tale analogia, o comparazione di uguaglianza, fra l'intellegibile e il sensibile è subito spiegata (3od), giacché Timeo precisa che la divinità ha prodotto « un vivente unico, visibile, che comprende in sé tutti i viventi che gli sono per na-

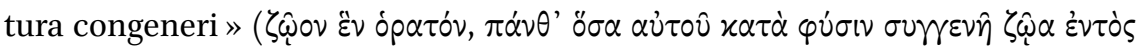

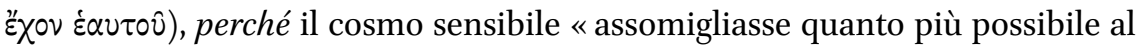

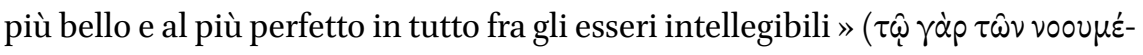

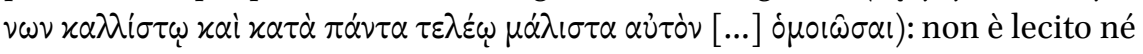
consigliabile concepire questa analogia in forma ascendente (ossia attribuendo al termine superiore caratteristiche che appartengono al termine inferiore e che da questo dunque gli deriverebbero), in modo che, se il cosmo sensibile è generato come un vivente in quanto dotato di anima e intelletto, allora anche il 
suo modello intellegibile deve essere rappresentato come un vivente in quanto a sua volta dotato di anima e intelletto; bensì, evidentemente, in forma discendente (ossia attribuendo al termine inferiore caratteristiche che appartengono al termine superiore e che da questo dunque gli derivano), riconoscendo perciò che, se il cosmo sensibile, generato come un vivente in quanto dotato di anima e intelletto, è una copia del modello intellegibile, allora il suo modello intellegibile deve essere rappresentato come un vivente in quanto a sua volta dotato di uno statuto e di una condizione "vitali", a qualche titolo superiore e in qualche senso eminente, che, al livello del sensibile, si manifestano nella forma e nei modi della "vita" sensibile, vale a dire nella presenza di un'anima dotata di intelletto innestata in un corpo che anima. Si giunge così al coerente compimento della rappresentazione dell'intellegibile come "vivente" (31a-b): se infatti, argomenta Timeo nel corso di una breve dimostrazione dell'unicità

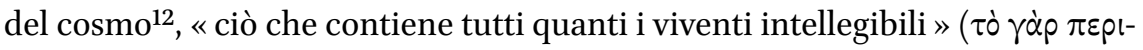

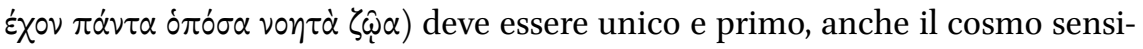
bile sarà uno solo, appunto in virtù della relazione di somiglianza, stabilita dal demiurgo, al «vivente perfetto » ( $\left.\tau \hat{\omega} \pi \alpha \nu \tau \varepsilon \lambda \varepsilon \hat{\imath} \zeta \omega_{\omega}^{\prime} \omega\right)$. Questa solenne dichiarazione, che implica l'esplicita denominazione di panteles zōion per qualificare l'intellegibile, appare riecheggiata più volte nel seguito del dialogo e nuovamente menzionata in $37 \mathrm{~d}$, ancora in riferimento all'obiettivo del demiurgo di rendere il cosmo sensibile quanto più possibile simile al suo modello, «che si

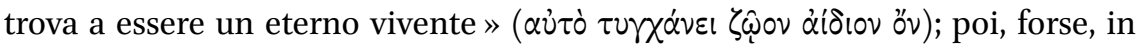
39e, quando viene evocata la capacità dell'intelletto di cogliere le specie che

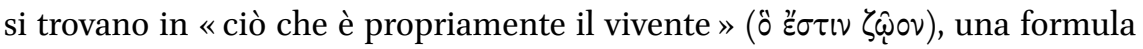
che designa abitualmente, nel lessico platonico, proprio la realtà delle idee ${ }^{13}$.

Quanto si può trarre da questi passi, attenendosi a una lettura rigorosa quanto prudente ed evitando brutali sovra-interpretazioni, è in primo luogo il carattere certamente analogico dell'attribuzione della "vita" e della "vitalità" all'intellegibile a partire dalla considerazione del sensibile: poiché il cosmo è un vivente sensibile ed è copia di un modello, allora il modello deve configurarsi come un vivente intellegibile. Ora, essere "vivente", per una realtà sensibile, significa disporre di un'anima che anima un corpo in cui è innestata, ma ciò pare valere appunto soltanto, come abbiamo visto poco sopra, nel caso del sensibile; cosa significa quindi, e cosa comporta, essere "vivente" per una realtà intellegibile? Non certo il possesso di un'anima, o di un intelletto (che

12 Cfr. soltanto su questo argomento Parry, «The unique world of the Timaeus »; e Patterson, «The unique worlds of the Timaeus».

13 Si veda in proposito Ferrari, «L'anima dell'essere. Sofista, 248 E-249 A e Timeo, 3o C-31 A», 6o8, n. 13 . 
non può sussistere se non in un'anima), perché, anche al di là di quanto già argomentato sulla base dei passi esaminati del Timeo (rispetto all'esigenza che l'anima e l'intelletto siano introdotti nel sensibile, precisamente per renderlo il più possibile simile alla perfezione del modello, che è invece privo di corpo sensibile e di per sé già perfetto), l'anima è in generale concepita da Platone, per esprimersi in modo molto sommario, secondo due tratti ontologici fondamentali. Essa ha innanzitutto natura e struttura intermedie fra il sensibile e l'intellegibile, sì da risultare composta tanto da elementi sensibili quanto da elementi intellegibili o da un mix di entrambi ${ }^{14}$, non lasciandosi così ricondurre interamente né all'ambito sensibile né all'ambito intellegibile, ma piuttosto rivelandosi in grado di esercitare, in virtù della sua funzione motrice, il governo del mondo sensibile e dei corpi, che a sua volta dipende dalla retta conoscenza dell'intellegibile che la sua funzione noetica le garantisce ${ }^{15}$. Quindi, e di conseguenza, l'anima intrattiene con l'intellegibile una relazione che non è evidentemente di identità, ma, come i dialoghi platonici spesso ripetono, di

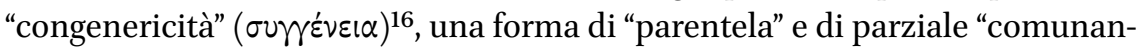
za", che le permette di accedere all'intellegibile e di giungere alla contemplazione delle idee ${ }^{17}$, il che ne attesta al di fuori di ogni dubbio la diversità da esse, giacché, se così non fosse e l'anima si rivelasse identica alle idee, non avrebbe senso porre il problema del suo accesso all'intellegibile né delle condizioni della sua contemplazione.

Ma torniamo così alla stessa conclusione già raggiunta in precedenza: se è privo di anima, non è certo in quanto "animato" che l'intellegibile può essere considerato un "vivente", perfetto o eterno; bisognerà dunque individuare per esso una condizione suscettibile di essere qualificata come "vitale", pur in assenza dell'anima, in quanto modello della condizione "vitale" che, nel sensibile, dipende invece dalla presenza dell'anima. Prima di compiere questo passo ulteriore, possiamo però constatare intanto che una simile conclusione porta a escludere una prima interpretazione, pure autorevolmente difesa, del panteles zōion del Timeo come propriamente dotato di un'anima o caratterizzato come un intelletto ${ }^{18}$. Non resta dunque che tentare di percorrere

\footnotetext{
14 Cfr. Tim. $34 \mathrm{c}-36 \mathrm{~d}$.

15 Cfr. Tim. $36 \mathrm{~d}-37 \mathrm{c}$.

16 Cfr. per esempio Men. 81c-d, Phd. 79b-d, Rep. x 611d-e.

17 Cfr. ancora Rep. x 611d-e e Tim. 42b; ma si veda pure il celebre mito di Phdr. 248a-c.

18 Posizione difesa, benché con sfumature diverse, da Krämer, Der Ursprung der Geistmetaphysik. Untersuchungen zur Geschichte des Platonismus zwischen Platon und Plotin, 194-201, e da Halfwassen, « Der Demiurg: seine Stellung in der Philosophie Platons und seine Deutung im antiken Platonismus », che tendono a concepire il panteles zōion del Timeo, in stretta relazione con il pantelōs on del passo del Sofista che passerò subito
} 
un'altra via che consenta cioè di comprendere altrimenti, vale a dire indipendentemente dall'implicazione di un'anima e di un intelletto, questa "vitalità" dell'intellegibile ${ }^{19}$.

Ora, se il Timeo non sembra offrire spunti in tale direzione, un passo particolarmente noto e controverso del Sofista appare invece piuttosto promettente. Si tratta di Soph. 248e-249a, che si colloca verso la conclusione della celebre $g i$ gantomachia fra i "nati dalla terra" e gli "amici delle idee", i primi che escludono dall'essere tutto ciò che non sia "corpo", i secondi che invece identificano ciò che è con certe "idee" immobili e immutabili: come possibile mediazione fra le due posizioni viene introdotta qui una nuova e diversa definizione dell'essere

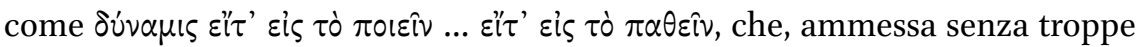
resistenze dai "materialisti", incontra invece la strenua opposizione degli "idealisti", consapevoli del fatto che, se accogliessero tale definizione dell'essere, si troverebbero pure costretti a riconoscere che le idee, realtà veramente essenti, risultano affette da un qualche genere di passione o azione $(\pi \dot{\alpha} \theta \eta \mu \alpha \hat{\eta}$

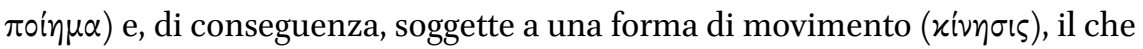
è impossibile secondo la loro dottrina, che impone che il movimento sia confinato all'ambito del divenire ${ }^{20}$. Si noti soltanto, fin d'ora, che il nodo intorno al quale ruota il confronto con gli "idealisti" consiste precisamente, e unicamente, nell'inclusione (cui li si vuole costringere) o nell'esclusione (cui essi si attengono accanitamente) della kinēsis, o di una sua qualche forma, nell'essere $(248 \mathrm{e})$; e non è certo un caso che l'esito di questo confronto, con la definitiva confutazione degli "idealisti" che esso comporta, preveda appunto, ancora una volta, l'unica ed esplicita ammissione loro estorta della kinēsis, o di una sua qualche forma, nell'essere (249b-d). Riproduco dunque il passo che segue e sul quale occorre adesso soffermarsi.

a esaminare, nella forma dell'identità organica di pensiero ed essere, vale a dire, secondo la celebre dottrina neoplatonica delle ipostasi, come essere "pensante" e "pensato" a un tempo; cfr. anche, in tale direzione e con particolare riferimento al Sofista, le vigorose osservazioni di Gerson, « The "Holy Solemnity" of Forms and the Platonic Interpretation of Sophist».

19 Così pure, giustamente, Ferrari, « L'anima dell'essere », 608.

20 Per quanto riguarda la traduzione e la comprensione di questa sezione del Sofista, che solleva notevoli problemi interpretativi, fin dalla ricostruzione delle diverse tappe della sua sequenza argomentativa, rinvio all'introduzione e alle note ad locum in Fronterotta, ed., Platone, Sofista, 75-89 e 370-379; ho invece particolarmente approfondito la natura e le implicazioni della definizione dell'essere come dynamis di agire e patire nei miei successivi studi « L'être et la participation de l'autre. Une nouvelle ontologie dans le Sophiste », e « La notion de $\triangle$ YNAMI $\Sigma$ dans le Sophiste de Platon: KOIN $\Omega$ NIA entre les formes et $\operatorname{ME} \Theta E \Xi I \Sigma$ du sensible à l'intelligible ». 


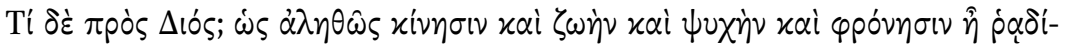

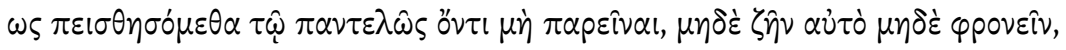

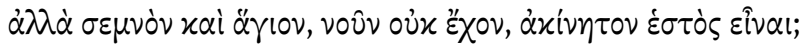

Ma allora, per Zeus? Ci lasceremo convincere senza colpo ferire che movimento, vita, anima e intelligenza davvero non siano presenti nella totalità dell'essere pieno, e che l'essere né viva né pensi, ma, venerabile $\mathrm{e}$ santo, se ne stia, privo d'intelletto, immobile e fermo?

Conviene limitare l'esame di questo passo, estremamente complesso e discusso, all'aspetto decisivo per i miei scopi attuali, vale a dire, come è ovvio, l'inclusione

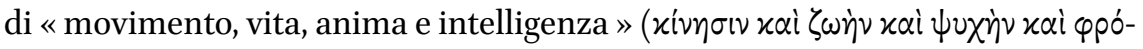

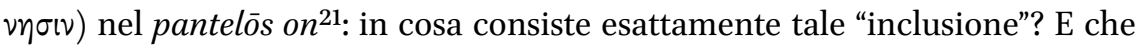
relazione ha, se ne ha una, con la "vitalità" attribuita all'intellegibile nel Timeo? Per rispondere a queste domande, bisogna innanzitutto prendere posizione sul significato del sintagma pantelōs on. Ė infatti possibile intendere l'avverbio pantelōs in senso estensivo, cioè con riferimento all'insieme" o alla "totalità" dell'essere e delle cose che sono, a designare così un ambito ontologico tanto esteso da comprendere allora sia il mondo sensibile sia la sfera intellegibile: in tal caso, l'inclusione di movimento, vita, anima e intelligenza nell'"insieme" o nella "totalità" dell'essere non si rivelerebbe affatto problematica, perché si potrebbe arguire, coerentemente con la posizione difesa dagli "idealisti", che

21 Fra gli aspetti più controversi, è decisivo per la comprensione del passo il significato da

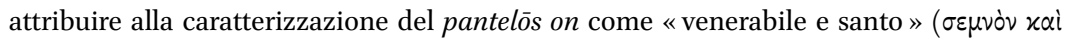
äriov), che si può intendere in almeno due modi diversi: (1) in senso concessivo, come tenderei a preferire $($ « $[\ldots]$ che l'essere né viva né pensi, ma, pur essendo venerabile e santo, se ne stia, privo d'intelletto, immobile e fermo [...]»), oppure (2) in senso causale («[...] che l'essere né viva né pensi, ma, in quanto venerabile e santo, se ne stia, privo d'intelletto, immobile e fermo [...]»). Nel primo caso, la "venerabilità" e la "santità" dell'essere dipenderebbero dalla sua "motilità" e "vitalità", sicché risulterebbe contraddittorio ammettere che esso «se ne stia [...] immobile e fermo », se è davvero «venerabile e santo » e dunque necessariamente "mobile"; nel secondo caso, invece, la "venerabilità" e la "santità" dell'essere sarebbero connesse alla sua "immobilità", sicché risulterebbe impossibile accettare che esso «venerabile e santo, se ne stia [...] immobile e fermo », perché occorre invece riconoscergli "motilità" e "vitalità". Questa scelta non è indifferente né, evidentemente, per la comprensione dell'argomento platonico né per la storia delle sue interpretazioni, da Aristotele a Plotino: si vedano ora in proposito Abbate, «Die dynamische und lebendige Natur des intelligiblen Seins bei Platon und in der neuplatonischen Überlieferung», Morel, «L'argomento della "venerabilità dell'essere" e la sua fortuna (Aristotele e Plotino, eredi di Platone, Sofista, 248e-249a) », e Fronterotta, « Movimento,

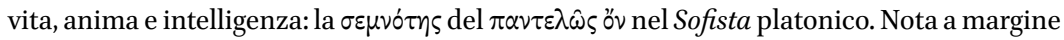
di P.-M. Morel, L'argomento della 'venerabilità dell'essere'e la sua fortuna ». 
movimento, vita, anima e intelligenza sono, sì, compresi nell' "insieme" o nella "totalità" delle cose che sono, ma confinati esclusivamente in quella parte del pantelōs on che coincide con la sua sezione sensibile, salvaguardando in tal modo l'assoluta immobilità della sua sezione intellegibile ${ }^{22}$. Vi è però da osservare, contro questa lettura, che il pantelōs on, pur rinviando a un ambito di realtà effettivamente plurale e comprensivo, non sembra tuttavia poter includere anche il mondo sensibile, e ciò per più ragioni. In primo luogo, il sintagma to pantelōs on deve essere certamente posto in contrapposizione a to mēdamōs on, evocato in $237 \mathrm{~b}$ : ora, poiché quest'ultimo si riferisce senza alcun dubbio al non essere assoluto, pare plausibile dedurne che il suo contrario, appunto to pantelōs on, indichi un ambito di realtà realmente e pienamente essente. Inoltre, in $249 \mathrm{~b}$, si afferma risolutamente che, se non si comprendesse il movimento fra le cose che sono, nel pantelōs on, e tutto fosse immobile (come vogliono gli "idealisti), non si darebbe nous «per nessuno, di nessuna cosa e in nessun modo »: ma nous è un termine che richiama abitualmente la vera conoscenza dell'essere in senso proprio, così distinguendosi da quella forma epistemica intermedia che coincide con l'opinione, rivolta esclusivamente alle cose sensibili. Infine, in $249 \mathrm{c}-\mathrm{d}$, si giunge alla conclusione che il "filosofo" deve ammettere «che entrambi gli ambiti, sia delle cose immobili sia di quelle in movimento, sono l'essere e il tutto »: ma, poco oltre (254a-b), il filosofo è descritto come colui il quale, nei suoi ragionamenti, si attiene sempre all'essere $(\tau \hat{\eta}$

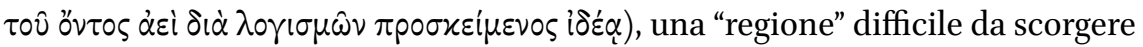

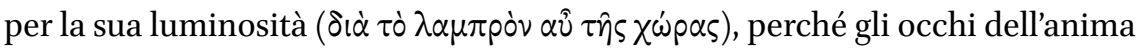
dei più sono incapaci di resistere alla vista della divinità ( $\tau \dot{\alpha} \gamma \dot{\alpha} \rho \tau \hat{\jmath} \varsigma \tau \hat{\omega} \nu \pi 0 \lambda \lambda \hat{\omega} \nu$

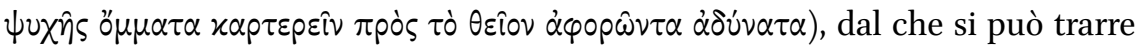
che l'oggetto della contemplazione del filosofo, che consiste nell'essere totale e comprensivo delle cose immobili come di quelle in movimento, altro non è che l'ambito ontologico più elevato, ossia appunto l'intellegibile soltanto.

Tutto ciò suggerisce pertanto di adottare una traduzione e un'interpretazione intensive del sintagma pantelōs on, da rendere dunque con "ciò che è realmente o pienamente" e da intendere in esclusivo riferimento all'intellegibile, tornando allora al problema di illustrare natura e modi dell'inclusione di movimento, vita, anima e intelligenza nell'essere in senso proprio, nell'intellegibile ${ }^{23}$. Sarei

22 Questa è l'interpretazione tradizionale del sintagma pantelōs on suggerita per esempio da Cornford, Plato's Theory of Knowledge, 245, e difesa oggi da Brisson, « La définition de l'être par la puissance. Un commentaire de Sophiste $247 \mathrm{~B}-249 \mathrm{D}$ ».

23 Adottano una traduzione e un'interpretazione intensive del sintagma pantelōs on, pur traendone poi conseguenze diverse, Gerson, «The 'Holy Solemnity' of Forms », 292, n. 3; Centrone, Platone, Sofista, xxxv-xxxix; Ferrari, «L'anima dell'essere», 6o2-6o4; e Morel, «L'argomento della "venerabilità dell'essere" ». Pur nel quadro di un'interpretazione 
tuttavia propenso a integrare questa opzione intensiva, che, per le ragioni appena esposte, considero più convincente, con una sfumatura estensiva che porti a riconoscere che il pantelōs on, senz'altro coincidente con l'intellegibile in senso stretto, implica però a un tempo una struttura plurale e organica, non certo tale da includere in sé il mondo sensibile, ma compatibile con un'articolazione dell'essere reale e pieno appunto in una pluralità organica di intellegibili: il significato complessivo del passo esaminato, del resto, non è tanto (o soltanto) quello di mostrare quanto sono movimento, vita, anima e intelligenza, bensì di collocarli nell'insieme o nella totalità delle cose che sono realmente e pienamente, il che è confermato oltre ogni dubbio dal fatto che l'inclusione di movimento, vita, anima e intelligenza nel pantelōs on è resa con il verbo pareinai, "essere presente" o "trovarsi compreso in", che esprime perciò la presenza di qualcosa in un luogo o, in questo contesto, in un ambito determinato di realtà, e non solo un grado maggiore o minore di intensione ontologica ${ }^{24}$. Comunque sia di ciò, come intendere a questo punto la "presenza" di movimento, vita, anima e intelligenza nel pantelōs on e quali implicazioni attribuirle? Ora, come ho chiarito in precedenza, vi sono a mio avviso decisivi argomenti, nei dialoghi platonici, che si oppongono alla possibilità di assegnare un'anima e un intelletto all'intellegibile, che si tratti del panteles zōion del Timeo o del pantelōs on del Sofista ${ }^{25}$, sicché conviene riprendere la questione a partire dal primo dei termini della sequenza costruita nel passo del Sofista, vale a dire dal movimento.

Già a questo livello infatti, e prima ancora di interrogarsi intorno all'anima e all'intelletto, emerge una difficoltà, giacché dall'introduzione del movimento nel pantelōs on parrebbe dover conseguire che questo ne sia affetto, contraddicendo così uno degli assunti più classici della concezione platonica dell'intellegibile come di per sé immobile e immutabile ${ }^{26}$. Non credo però, con buona parte dei commentatori ${ }^{27}$, che un simile esito sia inevitabile né,

intensiva del sintagma pantelōs on, si oppone tuttavia alla conclusione che l'argomento conduca a includere in esso movimento, vita, anima e intelligenza, Karfik, « Gott als Nous. Der Gottesbegriff Platons ».

24 In questa stessa direzione mi pare argomentare Abbate, « Die dynamische und lebendige Natur des intelligiblen Seins », 228.

25 Questa è invece, come già ricordato, la tesi di Gerson, « The 'Holy Solemnity' of Forms ».

26 Che le idee intellegibili finiscano, in ragione di questo argomento, per rivelarsi soggette al movimento, se non integralmente o in modo essenziale, almeno nella misura in cui vengono conosciute, e perciò mosse, dall'intelletto, è la conclusione di Moravcsik, « Being and meaning in the Sophist », 40, e di Bluck, Plato's Sophist. A Commentary, 96-10o.

27 Fra quanti hanno negato recisamente la possibilità di ascrivere a Platone un mutamento di prospettiva così radicale nella propria concezione dell'intellegibile, che attribuirebbe alle idee e all'essere tratti di mobilità e di mutamento, vanno ricordati soprattutto Cornford, Plato's Theory of Knowledge, 244-248, Cherniss, The Riddle of the Early 
d'altro canto, suggerito dal testo: tendo a pensare piuttosto che l'introduzione del movimento nel pantelōs on e la relazione così stabilita fra il movimento e l'intellegibile, due acquisizioni teoriche che si ricavano indubbiamente da questo passo del Sofista, si lascino spiegare senza compromettere la tesi dell'immobilità e dell'immutabilità dell'essere, ma semplicemente tenendo conto della differenza ontologica che sussiste fra il mondo sensibile e l'ambito intellegibile. Così come nel caso di qualunque proprietà sensibile rispetto all'idea intellegibile corrispondente si constata che la prima manifesta concretamente ed effettivamente i tratti di cui è la proprietà, mentre la seconda non esprime che l'essenza o la forma di quella proprietà - la molteplicità, per esempio, si manifesta sul piano sensibile sotto l'aspetto di una concreta ed effettiva pluralità di enti, laddove, sul piano intellegibile, essa si pone come essenza o forma della molteplicità senza essere per questo più di una, se è vero che l'idea della molteplicità è in sé appunto una e non molteplice - analogamente, per quanto riguarda il movimento, si potrà sostenere che, a differenza della sua "versione" sensibile, la kinēsis dell'intellegibile e nell'intellegibile non consiste in un concreto mutamento di luogo o di aspetto né tantomeno in un'effettiva alterazione, ma soltanto in una condizione a qualche titolo dinamica che sia compatibile con i requisiti ontologici dell'immobilità e dell'immutabilità che devono appartenere per definizione all'intellegibile. Per capire di quale condizione precisamente si tratti, e in cosa consista il suo dinamismo, si può forse prestare attenzione agli altri tre termini della sequenza presentata nel passo del Sofista, ossia "vita", "anima" e "intelligenza", da intendere a questo punto

Academy, 81, e Vlastos, «An Ambiguity in the Sophist », Appendix I: On the Interpretation of Sph. 248d4-e4, 309-317. A sostegno di questa posizione si può ricordare che Euthyphr.

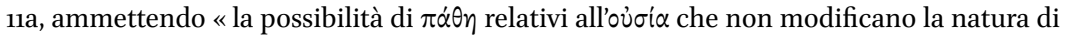

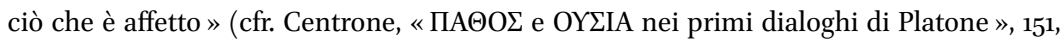
n. 45), permetterebbe di sostenere che le idee costituiscono l'oggetto della conoscenza, e in tal senso subiscano un pathos, senza che ciò imponga loro necessariamente di essere mosse. Una tesi diversa sostiene De Rijk, Plato's Sophist. A Philosophical Commentary, 105-109, che capovolge la prospettiva di lettura: non si tratta in effetti di chiedersi se le idee si muovano, ma di riconoscere che il movimento è assunto, accanto alle idee e fra le idee, nell'intellegibile. Ciò non toglie, tuttavia, che ci si interroghi sulle conseguenze di questo passo: una volta introdotto il movimento nell'intellegibile, ne seguirà che le idee si muovano? A tale ragionevole questione, De Rijk risponde suggerendo una distinzione in base alla quale, come enti separati e trascendenti, le idee rimangono del tutto pure e immobili, mentre invece, in quanto soggette alla partecipazione da parte dei sensibili, entrano in questa misura in contatto con le realtà in divenire, subendo un'affezione che le pone in movimento. Personalmente, ritengo implausibile una simile distinzione, se non altro perché qui nessun accenno è rivolto al problema della partecipazione delle cose in divenire alle idee intellegibili né la realtà sensibile tout court sembra in nessun modo chiamata in causa. 
piuttosto come esplicativi del primo, del movimento, che non come altrettanti caratteri supplementari da aggiungere nel pantelōs on.

Che ciò sia possibile, e anzi probabile, si ricava fra l'altro da quanto già osservato in relazione alla pressoché esclusiva centralità della kinēsis nel corso dell'esame e della confutazione della posizione degli "idealisti": la proposta di una nuova definizione dell'essere come dynamis di agire e patire $(247 \mathrm{~d}-\mathrm{e})$ ha proprio il fine di costringere gli "idealisti" ad ammettere che l'essere non è completamente immobile e inerte e, per questa ragione, è da essi respinta (248e); e anche la conclusione dell'esame comporta sostanzialmente l'unico risultato di accogliere il movimento nell'essere e, per conseguenza, di abbandonare la posizione degli "idealisti", facendo cadere così, implicitamente, la loro opposizione alla definizione dell'essere come dynamis di agire e patire $(249 \mathrm{~b}-\mathrm{d})$. Ritengo inoltre che il testo stesso del nostro passo, in $248 \mathrm{e}-249 \mathrm{a}$, si lasci interpretare in questo senso, solo che si legga la sequenza xivnolv xai

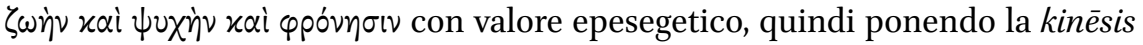
e la sua inclusione nel pantelōs on quale fulcro della dimostrazione e i termini $z \bar{o} \bar{e}, p s y c h \bar{e}$ e phronēsis come sue specificazioni, appunto per precisare il tipo di movimento di cui si stabilisce l'inclusione nell'essere ${ }^{28}$. Il passo potrebbe allora essere parafrasato come segue: "Ci lasceremo convincere senza colpo ferire che il movimento, cioè quel movimento vitale che è dello stesso genere di quello prodotto dall'attività dell'anima e che corrisponde alla funzione noetica dell'intelletto, davvero non sia presente nella totalità dell'essere pieno, e che l'essere non possieda tratti vitali né intellettuali, ma, venerabile e santo, se ne stia, privo di carattere noetico, immobile e fermo?" In tal caso, come si vede, ci troveremmo di fronte a una risposta coerente alla questione sollevata poco sopra: a differenza del movimento sensibile, che implica un concreto mutamento di luogo o un'effettiva alterazione di stato, la kinēsis dell'intellegibile e nell'intellegibile coincide con un dinamismo che può essere detto "vitale" in quanto omogeneo a quello prodotto dall'anima, e dunque sottoposto a un rigoroso criterio di ordine e regolarità che non suppone alcun movimento nello spazio, e che si svolge in termini esclusivamente noetici o intellettuali, e quindi indipendentemente da ogni connessione con la materia e i corpi e dal mutamento a essi intrinseco. Si tratterebbe insomma di riconoscere al pantelōs on un movimento "vitale" analogo a quello dell'anima, ma che non implica la presenza dell'anima, e un carattere "dinamico" analogo a quello dell'intelletto, ma che non implica la presenza dellintelletto, senza che, in altre parole, questa

28 Sullintima connessione fra "movimento", "vita" e "anima" (e "intelligenza"), che rappresenta una costante nella riflessione di Platone, si veda ancora Fronterotta, ed., Platone, Timeo, $72-74$. 
duplice analogia conduca all'estrema e ai miei occhi insostenibile conclusione che il pantelōs on sia effettivamente "animato" e "pensi".

Di una simile prospettiva esegetica mi spingerei infine a tratteggiare l'esito ultimo, formulando l'ipotesi, che andrebbe certo più adeguatamente fondata, ma di cui non posso dare conto qui estesamente, secondo la quale il movimento "vitale" del pantelōs on evocato in questo passo e fatto oggetto di analisi altro non riveli che il concreto sviluppo della definizione dell'essere come $d y$ namis di agire e patire, che, richiamata nel seguito del dialogo nella forma di una dynamis koinōnias (in 251e; oppure dynamis epikoinōnias in 252d), si traduce allora nella capacità, di cui gli intellegibili dispongono e in cui consistono propriamente il loro essere e la loro definizione, di stabilire reciproci rapporti di "comunicazione" o "partecipazione" 29 . La kinēsis dell'essere, assimilata a un tratto psichico o noetico, andrebbe a questo punto concepita come l'insieme di movimenti, prodotti o subiti, che pongono gli intellegibili in reciproca koinōnia - "comunicare" equivalendo a un'azione, e pertanto a un movimento prodotto che discende da una dynamis di agire, ed "essere comunicato" equivalendo a una passione, e pertanto a un movimento subito che discende da una dynamis di patire -, la "vitalità" del pantelōs on manifestandosi quindi senza residui nella sua dimensione dinamico-relazionale interna ${ }^{30}$.

Mi pare che questa interpretazione, pur basata su elementi che sono in parte inevitabilmente congetturali, si presenti come la più coerente (o la meno incoerente) per una comprensione non aporetica del passo esaminato del Sofista e del suo contesto argomentativo, ma anche, derivativamente, per una spiegazione efficace dell'altrimenti oscura rappresentazione dell'intellegibile come un panteles zōon nel Timeo, rendendo conto in entrambi i casi della natura dinamica e della struttura organica dell'essere nella sua funzione di modello della realtà sensibile plurale e diveniente ${ }^{31}$.

29 Per l'interpretazione della definizione dell'essere come dynamis di agire e patire (249b-d) e i suoi successivi sviluppi, nel Sofista, come dynamis koinōnias e dunque come chiave di volta per la comprensione della sezione ontologica del dialogo dedicata alla koinōnia tōn genōn, rinvio nuovamente ai miei studi «L'être et la participation de l'autre », e « La notion de $\triangle$ YNAMI $\Sigma »$.

30 A una conclusione appena meno esplicita di quella da me tratteggiata giungono Centrone (ed.), Platone, Sofista, xxxix, e, più prudentemente, Morel, «L'argomento della "venerabilità dell'essere" ». Non mi è chiaro invece se Abbate, «Die dynamische und lebendige Natur», accolga anch'egli un'interpretazione soltanto dinamico-relazionale della "vitalità" dell'intellegibile o se invece intenda attribuire a quest'ultimo una dimensione propriamente psichica e noetica, come parrebbe suggerito dal suo richiamo alla lettura plotiniana del passo del Sofista.

31 Diversamente intende Ferrari, L'anima dell'essere, 6o8-613, che, ricollegandosi alla sua interpretazione della figura e della funzione del demiurgo (cfr. supra, n. 4), considera la 


\section{Works Cited}

Abbate, Michele. «Die dynamische und lebendige Natur des intelligiblen Seins bei Platon und in der neuplatonischen Überlieferung». In Selbstbewegung und Lebendigkeit. Die Seele in Platons Spätwerk. A cura di Michele Abbate, Julia Pfefferkorn e Antonino Spinelli. Berlin: De Gruyter, 2016, 227-242.

Algra, Keimpe. Concepts of Space in Greek Thought. Leiden: Brill, 1995.

Bluck, R. S. Plato's Sophist. A Commentary. Manchester: Manchester University Press, 1975 .

Brisson, Luc. Le Même et l'Autre dans la structure ontologique du Timée de Platon. Terza edizione. Sankt Augustin: Academia Verlag, 1998.

Brisson, Luc. «La notion de phtónos chez Platon». Ora in Lectures de Platon. Vrin, Paris 200o, 219-234.

Brisson, Luc. «La définition de l'être par la puissance. Un commentaire de Sophiste $247 \mathrm{~B}-249 \mathrm{D} »$ In $\triangle \mathrm{YNAMI}$. Autour de la puissance chez Aristote. A cura di Michel Crubellier, Annick Jaulin, David Lefebvre, e Pierre-Marie Morel. Louvain-La-Neuve: Peeters, 2008, 173-186.

Brisson, Luc. «La matière chez Platon et dans la tradition platonicienne». In Materia, XIII Colloquio Internazionale, Roma, 7-9 gennaio 2010. A cura di Delfina Giovannozzi e Marco Veneziani. Firenze: Olschki, 2011, 1-40.

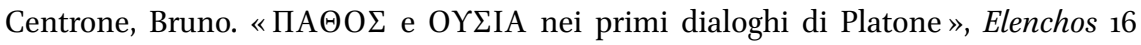
(1995): 131-152.

Centrone, Bruno, ed. Platone, Sofista. Torino: Einaudi, 2008.

Cherniss, H. F. The Riddle of the Early Academy. Princeton: Princeton University Press, 1945 .

Cornford, Francis. Plato's Theory of Knowledge. The Theaetetus and the Sophist of Plato. Translated with a running commentary. London: Routledge \& Kegan Paul, 1935.

De Rijk, L. M. Plato's Sophist. A Philosophical Commentary. Amsterdam: North-Holland Publishing Company, 1986.

Donini, Pier Luigi. «Il Timeo: unità del dialogo, verosimiglianza del discorso». Elenchos 9 (1988): 5-52.

"vitalità" del panteles zōon del Timeo come l'espressione della sua funzione causale efficiente nei confronti del cosmo sensibile, per estendere retrospettivamente questa lettura al pantelōs on del Sofista, cui non può però che attribuire un'azione causale interna all'intellegibile stesso (non troppo dissimile da quella da me prefigurata sopra). Anche al di là delle mie perplessità sulla prima parte di questa opzione esegetica (cfr. ancora supra, n. 4), ritengo poco plausibile che la "vitalità" del panteles zōon e del pantelōs on si esplichi in forme, modi e contesti tanto distanti da apparire irriducibili a una comprensione unitaria e omogenea. 
Ferrari, Franco. « Questioni eidetiche ». Elenchos 24 (2003): 93-113.

Ferrari, Franco. « Causa paradigmatica e causa efficiente: il ruolo delle idee nel Timeo ». In Plato physicus. Cosmologia e antropologia nel Timeo. A cura di Carlo Natali e Stefano Maso. Amsterdam: Hakkert, 2003, 83-96.

Ferrari, Franco. «La chora nel Timeo di Platone. Riflessioni su "materia" e "spazio" nell'ontologia del mondo fenomenico ». Quaestio 7 (2007): 3-23.

Ferrari, Franco. «Der entmythologisierte Demiurg». In Platon und das Göttliche. A cura di Dietmar Koch, Irmegard Männlein-Robert e Niels Weidtmann. Tübingen: Attempto Verlag, 2010, 62-81.

Ferrari, Franco. «L'anima dell'essere. Sofista, 248 E-249 A e Timeo, 3 o C-31 A ». In Logon didonai. La filosofia come esercizio del rendere ragione. Studi in onore di Giovanni Casertano. A cura di Lidia Palumbo. Napoli: Loffredo, 2012, 6o1-613.

Fronterotta, Francesco. «L'être et la participation de l'autre. Une nouvelle ontologie dans le Sophiste ». Les Etudes Philosophiques (1995/3): 311-353.

Fronterotta, Francesco. ME $\mathrm{EEI}$. La teoria delle idee e la partecipazione delle cose empiriche. Dai dialoghi giovanili al Parmenide. Pisa: Edizioni della Scuola Normale Superiore, 2001.

Fronterotta, Francesco. «Questioni eidetiche in Platone: il sensibile e il demiurgo, l'essere e il bene ». In Giornale critico della filosofia italiana LXXXV (2006): 412-436.

Fronterotta, Francesco, ed. Platone, Sofista. Milano: Rizzoli, 2007.

Fronterotta, Francesco. «La notion de $\triangle$ YNAMI $\Sigma$ dans le Sophiste de Platon:

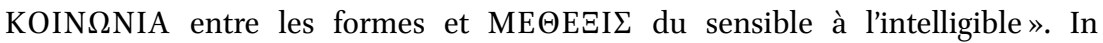
$\Delta$ YNAMI $\Sigma$. Autour de la puissance chez Aristote. A cura di Michel Crubellier, Annick Jaulin, David Lefebvre, e Pierre-Marie Morel. Louvain-La-Neuve: Peeters, 20o8, $187-224$.

Fronterotta, Francesco. «Chiusura causale della fisica e razionalità del tutto: alcune opzioni esegetiche sull'efficienza causale delle idee platoniche ». Plato. The Internet Journal of the International Plato Society (8): 2008, http://gramata.univ-parisı.fr/ Plato/.

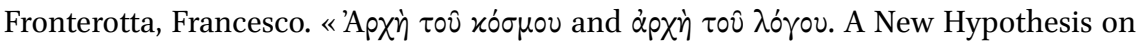
the Beginning of the World in Plato's Timaeus ». In Philosophy and Dialogue. Studies on Plato's Dialogues. A cura di Antoni Bosch-Veciana and Josep Monserrat-Molas. Barcelona: Barcelonesa d'Edicions, 2010, 141-155.

Fronterotta, Francesco, ed. Platone, Timeo. Terza edizione. Milano: Rizzoli, 2011.

Fronterotta, Francesco. «Luogo, spazio e sostrato "spazio-materiale" nel Timeo di Platone e nei commenti al Timeo ». In Locus-Spatium. XIV Colloquio Internazionale, Roma, 3-5 gennaio 2013. A cura di Delfina Giovannozzi e Marco Veneziani. Firenze: Olschki, 2014, 7-42.

Fronterotta, Francesco. «Modello, copia, ricettacolo: monismo, dualismo o triade di principi nel Timeo?» Méthexis, XXVII (2014): 95-118. 


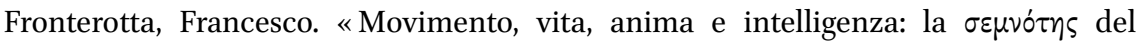
$\pi \alpha \nu \tau \varepsilon \lambda \hat{\omega} \varsigma$ oैv nel Sofista platonico. Nota a margine di P.-M. Morel, L'argomento della 'venerabilità dell'essere' e la sua fortuna ». Antiquorum Philosophia 12 (2018): 27-36.

Gerson, Lloyd. "The "Holy Solemnity" of Forms and the Platonic Interpretation of Sophist». Ancient Philosophy 26 (2006): 291-304.

Halfwassen, Jens. « Der Demiurg: seine Stellung in der Philosophie Platons und seine Deutung im antiken Platonismus ». In Le Timée de Platon. Contribution à l'histoire de sa réception. Platos Timaios. Beiträge zu seiner Rezeptionsgeschicht. A cura di Ada Neschke-Hentschke. Louvain: Peeters, 200o, 39-62.

Karfík, Filip. «Gott als Nous. Der Gottesbegriff Platons ». In Platon und das Göttliche. A cura di Dietmar Koch, Irmegard Männlein-Robert e Niels Weidtmann. Tübingen: Attempto Verlag, 2010, 82-97.

Krämer, Hans-Joachim. Der Ursprung der Geistmetaphysik. Untersuchungen zur Geschichte des Platonismus zwischen Platon und Plotin. Seconda edizione. Amsterdam: Grüner, 1967 .

Leszl, Walter. «Ragioni per postulare idee». In Eidos-Idea. Platone, Aristotele e la tradizione platonica. A cura di Francesco Fronterotta e Walter Leszl. Sankt Augustin: Academia Verlag, 2005, 37-74.

Miller, Dana. The Third Kind in Plato's Timaeus. Göttingen: Vandenhoeck \& Ruprecht, 2003.

Moravcsik, J. M. E. « Being and meaning in the Sophist». Acta Philosophica Fennica 14 (1962): 23-78.

Morel, Pierre-Marie. «L'argomento della "venerabilità dell'essere" e la sua fortuna (Aristotele e Plotino, eredi di Platone, Sofista, 248e-249a)». Antiquorum Philosophia 12 (2018): 11-26.

Natali, Carlo. «La forma platonica è una causa formale? » In Platon und Aristoteles sub ratione veritatis. Festschrift für W. Wieland zum 7o. Geburtstag. A cura di Gregor Damschen, Rainer Enskat e Alejandro Vigo. Göttingen: Vandenhoeck \& Ruprecht, 2003, 158-173.

Parry, R. D. «The unique world of the Timaeus ». Journal of the History of Philosophy 17 (1979): 1-10.

Patterson, R. A. «The unique worlds of the Timaeus ». Phoenix, 35 (1981): 105-119.

Perl, Eric. «The Demiurge and the Forms. A Return to the Ancient Interpretation of Plato's Timaeus ». Ancient Philosophy 18 (1998): 81-92.

Sedley, David. «Platonic Causes ». Phronesis 43 (1998): 114-132.

Vlastos, Gregory. «An Ambiguity in the Sophist». In Platonic Studies. Princeton: Princeton University Press, 1973, 270-317. 